\title{
GAUGE INVARIANCE IN 2PI EFFECTIVE ACTIONS
}

\author{
E. MOTTOLA \\ Theoretical Division, T-8 \\ MS B285, \\ Los Alamos National Laboratory \\ Los Alamos, NM 87545 \\ E-mail: emil@lanl.gov
}

\begin{abstract}
The problem of maintaining gauge invariance in the 2PI formulation of QED is discussed. A modified form of the 2PI effective action is suggested in which Ward identities for external (background field) and internal (quantum field) gauge transformations are both satisfied, but in different manners. The residual gauge-fixing dependence in this modified $2 \mathrm{PI}$ formulation vanishes in a certain low momentum limit, which allows it to be used reliably for calculating quantities such as transport coeffcients and soft field relaxation in hot gauge theories.
\end{abstract}

The 1PI (one particle irreducible) effective action is the standard form of the effective action, which is a functional of classical field variables whose values may be regarded as expectation values of the underlying quantum fields. The first variation of the 1PI effective action produces equations of motion for these expectation values. Although sufficient in principle for most purposes, in practice the 1PI effective action is easily evaluated only in some approximation scheme, such as a loop expansion, Hartree or large $N$ expansion, ${ }^{1}$ which limits its range of applicability. In particular, these simplest loop or mean field approximations are incapable of describing collisional relaxation processes in hot or dense non-equilibrium plasmas. A consistent treatment of the effects of the hard quasi-particle degrees of freedom in gauge theories on the more slowly varying mean fields, even to leading order in the gauge coupling, is an outstanding challenge for non-equilibrium field theory. Yet a consistent formulation with gauge fields is just what is required in many applications, such as in formation and expansion of the quark-gluon plasma produced in an ultra-relativistic heavy ion collision, or in baryogenesis in a first order electroweak phase transition. Systematic improvements of the QCD equation of state beyond perturbation theory will also require such a consistent resummation scheme. 
The 2PI (two particle irreducible) effective action is a general field theoretic tool for addressing this problem. ${ }^{2,3}$ In constructing it one introduces sources for both the fields and their two-point bilinears. The Legendre transform with respect to this extended set of sources results in an effective action that is a functional of both mean fields and their two-point correlation functions, treated on the same footing. The 2PI formulation, sometimes known also as the ' $\Phi$-derivable' effective action has the advantage of being thermodynamically consistent, in the sense that any extremum of the $2 \mathrm{PI}$ free energy is automatically a minimum if the underlying quantum Hamiltonian is bounded from below. An arbitrary truncation scheme of $n$-point functions need not preserve this property in general. Although the 2PI effective action becomes equal to the 1PI effective action when evaluated at its exact extremum, somewhat different and more efficient expansion methods suggest themselves in the 2PI formulation. For example the proper self-energy, needed for evaluating collisional and damping effects, and which connects the Boltzmann description with the field theoretic one may be derived from the $2 \mathrm{PI}$ effective action from only the first few non-vanishing two-loop skeleton diagrams. ${ }^{4}$ This corresponds to a quite non-trivial resummation of an infinite class of perturbative diagrams beyond the leading order large $N$ or Hartree approximations.

When the mean fields are gauge fields we face an additional problem. The 2PI effective action runs into trouble in its simplest form, since it concentrates on resumming two-point correlation functions but ignores vertex corrections, which must accompany those two-point functions in a gauge theory, in order for Ward identities to be satisfied. In this contribution I show that the simplest two-loop approximation to the 2PI effective action in the simplest gauge theory, i.e. electromagnetism suffers from this problem, but that the gauge non-invariance of a slightly modified 2PI formulation vanishes in a certain kinematic limit relevant for relaxational processes.

If we apply the usual 2PI recipe to ordinary QED, treating the electron propagator $\mathcal{G}$ and photon propagator $\mathcal{D}_{\mu \nu}$ on exactly the same footing then we arrive at

$$
\begin{aligned}
& \mathcal{S}_{2 P I}[A ; \mathcal{G}, \mathcal{D}]=S_{c l}[A]-i \operatorname{Tr} \ln \mathcal{G}^{-1}+\frac{i}{2} \operatorname{Tr} \ln \mathcal{D}^{-1} \\
& \quad-i \operatorname{Tr}\left(G^{-1}[A] \cdot \mathcal{G}-1\right)+i \operatorname{Tr}\left(d^{-1} \cdot \mathcal{D}-1\right)+\Phi_{2}[\mathcal{G}, \mathcal{D}]
\end{aligned}
$$

The classical action $S_{c l}[A]$ is the Maxwell action and the lowest order inverse propagators for the electron and photon are defined by

$$
G^{-1}[A] \equiv i\left(\gamma^{\mu} \partial_{\mu}-i \gamma^{\mu} A_{\mu}+m\right)
$$




$$
d_{\mu \nu}^{-1} \equiv-\frac{\delta^{2} S_{c l}}{\delta A^{\mu} \delta A^{\nu}}=-\frac{1}{e^{2}}\left(\eta_{\mu \nu} \square-\partial_{\mu} \partial_{\nu}\right)
$$

The functional (1) is perfectly gauge invariant under background field gauge transformations,

$$
A_{\mu} \rightarrow A_{\mu}+\partial_{\mu} \theta
$$

provided that the both the lowest order and exact electron inverse Green's functions transform as

$$
\mathcal{G}^{-1} \rightarrow e^{-i \theta} \mathcal{G}^{-1} e^{i \theta}
$$

and the functional $\Phi_{2}$ involves only traces. For example if $\Phi_{2}$ is given by the lowest order non-trivial 2PI two-loop expression,

$$
\Phi_{2}[\mathcal{G}, \mathcal{D}]=\frac{1}{2} \int d^{4} x d^{4} x^{\prime} \mathcal{D}_{\mu \nu}\left(x, x^{\prime}\right) \operatorname{tr}\left\{\mathcal{G}\left(x, x^{\prime}\right) \gamma^{\mu} \mathcal{G}\left(x^{\prime}, x\right) \gamma^{\nu}\right\}
$$

then invariance under (5) is assured by the cyclic property of the trace.

Despite this invariance under background field gauge transformations it is easy to see that the $2 \mathrm{PI}$ effective action with $\Phi_{2}$ given by (6) cannot be a fully satisfactory approximation to QED. The reason is that the photon self-energy derived from $\Phi_{2}$, namely,

$$
i \operatorname{tr}\left\{\mathcal{G}\left(x, x^{\prime}\right) \gamma_{\mu} \mathcal{G}\left(x^{\prime}, x\right) \gamma_{\nu}\right\}
$$

is not transverse, in general, if $\mathcal{G} \neq G[A]$. Transversality of the photon self-energy depends upon the Ward-Takahashi identity,

$$
\left(p_{1}-p_{2}\right)_{\mu} \Gamma^{\mu}\left(p_{1}, p_{2}\right)=\mathcal{G}^{-1}\left(p_{1}\right)-\mathcal{G}^{-1}\left(p_{2}\right)
$$

between the photon-fermion vertex $\Gamma^{\mu}$ and the inverse fermion propagators in the exact theory. Since we have a resummed Green's function in the 2PI effective action but only the bare vertex function, $\Gamma^{\mu}\left(p_{1}, p_{2}\right)=\gamma^{\mu}$, the identity (8) is not satisfied, and the polarization (7) is not transverse.

The problem is that although the $2 \mathrm{PI}$ representation (1) is exact, any truncation scheme for $\Phi_{2}$ in terms of skeleton diagrams involving $\mathcal{G}$ and $\mathcal{D}$ leads to equations for these propagators that sum only a certain subset of higher loop diagrams with a fixed topology, determined by the choice of $\Phi_{2}$. However, the exact identity (8) requires cancellations at a given loop order between diagrams of different topology, not included in the same subset. This non-invariance with respect to gauge transformations on internal lines remains in any $2 \mathrm{PI}$ truncation scheme, nothwithstanding full invariance with respect to external gauge fields $A_{\mu}$, which is guaranteed by the general background field method. The difference between the Ward identities 
satisfied by the expectation values and those typically not satisfied by the internal propagators and vertices can be seen even in a non-gauge $O(N)$ scalar $\phi^{4}$ theory, where the failure of Goldstone's theorem for the internal propagators was noted some time ago. ${ }^{5}$

This problem may not be as severe as it appears at first sight. After all, we are hoping to use any truncation scheme in the $2 \mathrm{PI}$ formalism to calculate some quantities only to a given order in weak coupling parameter. If we can show that the errors we make by not having a strictly gauge invariant formulation are higher order in the coupling for the quantities of interest, the truncation scheme based on the 2PI effective action would still be useful and reliable to this order. Adopting such a strategy also might make possible modifications of the original 2PI formalism by neglecting some higher order contributions from the very beginning.

One such modification, suggested to restore the transversality of the photon self-energy, is to replace the resummed polarization (7) by the lower order form,

$$
\Pi_{\mu \nu}\left(x, x^{\prime}\right)=i \operatorname{tr}\left\{G\left(x, x^{\prime}\right) \gamma_{\mu} G\left(x^{\prime}, x\right) \gamma_{\nu}\right\},
$$

so that the photon inverse propagator becomes

$$
\mathcal{D}_{\mu \nu}^{-1} \rightarrow D_{\mu \nu}^{-1} \equiv d_{\mu \nu}^{-1}+\Pi_{\mu \nu} .
$$

Since this is nothing but the photon inverse propagator in the leading order of the large $N_{f}$ expansion, it is certainly transverse. Using the corresponding propagator $D_{\mu \nu}$ instead of $\mathcal{D}_{\mu \nu}$ everywhere in (1) and (6) results in the following form for the fermion inverse propagator and self-energy,

$$
\mathcal{G}^{-1}=G^{-1}+\Sigma=G^{-1}-i \gamma^{\mu} \mathcal{G} \gamma^{\nu} D_{\mu \nu},
$$

which is still a non-trivial Schwinger-Dyson resummation for $\mathcal{G}$ beyond leading order. Varying this formula with respect to the background gauge field $A_{\mu}$, one obtains a non-trivial Schwinger-Dyson equation for the vertex,

$$
\Gamma^{\mu} \equiv \frac{\delta \mathcal{G}^{-1}}{\delta A_{\mu}}=\gamma^{\mu}+i\left(\gamma^{\alpha} \mathcal{G} \Gamma^{\mu} \mathcal{G} \gamma^{\beta}\right) D_{\alpha \beta},
$$

which generates a sum over ladder diagrams. Because this vertex function is defined by varying $\mathcal{G}^{-1}$ with respect to the background field, it obeys the Ward identity (8). In this way we may modify the original 2PI effective action to satisfy the Ward identities for both the internal vertex $\gamma^{\mu}$, and the external resummed vertex $\Gamma^{\mu}$, albeit in different ways.

It might seem that we have avoided the gauge non-invariance problem entirely by this method. This is not the case, however, because gauge 
invariance also requires that in inverting the transverse $D_{\mu \nu}^{-1}$ of (10) to find the photon propagator $D_{\mu \nu}$ to appear in (11), we should be allowed to add an arbitrary longitudinal part to $D_{\mu \nu}$ without affecting the results. Because the self-energy $\Sigma$ in (11) involves a resummed fermion propagator $\mathcal{G}$ but only the bare vertex $\gamma^{\mu}$, one can see fairly easily that the usual proof that these longitudinal gauge dependent pieces of $D_{\mu \nu}$ drop out will not go through. In other words, the fermion self-energy is still gauge-fixing dependent. ${ }^{6}$ It is again higher loop diagrams with a different topology (crossed rainbows) that are required, but which are not included in the resummation of rainbow diagrams given by (11).

If one does go through the usual proof of gauge fixing independence, one finds that the non-cancellation within the rainbow approximation generated by (11) vanishes in the limit that the fermion momentum $p$ is much larger than the photon momentum $k$. Indeed the non-cancellation is of order $\left|\frac{k^{2}}{p^{2}}\right|$. Hence observables sensitive only to the kinematic range of momenta $\left|k^{2}\right| \ll\left|p^{2}\right|$ may be reliably computed by the modified 2PI scheme, and are gauge invariant up to corrections of this order. This kinematic regime of soft photon exchange is precisely that which determines the zero frequency electrical conductivity of the high temperature QED plasma. ${ }^{7}$ It is also the kinematic region of interest in the gap equation for the fermions in high density QCD. ${ }^{8}$ Further, by including higher order skeleton diagrams in $\Phi_{2}$ in this modified 2PI formulation one may systematically suppress the

residual gauge dependence to higher orders in $\left|\frac{k^{2}}{p^{2}}\right|$. A proof of these bounds and application to relaxational problems in non-equilibrium gauge theories will be presented elsewhere.

This research is supported by the Department of Energy, under contract $W$-7405-ENG-36 and is LANL Technical Report, LA-UR-03-2804.

\section{References}

1. See e.g. F. Cooper, S. Habib, Y. Kluger and E, Mottola, Phys. Rev. D55, 6471 (1997), and references therein.

2. J. M. Luttinger and J. C. Ward, Phys. Rev. 118, 1417 (1960).

3. J. M. Cornwall, R. Jackiw and E. T. Tomboulis, Phys. Rev. D10, 2428 (1974).

4. G. Baym and L. P. Kadanoff, Phys. Rev. 124, 287 (1961); G. Baym, Phys. Rev. 127, 1391 (1962).

5. G. Baym and G. Grinstein, Phys. Rev. D15, 2897 (1977).

6. A. Arrizabalaga and J. Smit, Phys. Rev. D66, 065014 (2002).

7. S. Jeon, Phys. Rev. D47, 4586 (1993); 52, 3591 (1995); S. Jeon and L. G. Yaffe, Phys. Rev. D53, 5799 (1996).

8. R. D. Pisarski and D. H. Rischke, Nucl. Phys. A702, 177 (2002). 